\title{
Trading Activity, Quoted Liquidity, and Stock Volatility*
}

\author{
Li Jiang \\ Hong Kong Baptist University, Hong Kong \\ Lawrence Kryzanowski \\ Concordia University, Canada
}

In this article, we examine dynamic relationships between volatility and various microstructure measures of trade activity and quoted liquidity for each component stock in the Toronto Stock Exchange 35 Index and for the Toronto 35 Index Participation Shares. When volatility is conditioned on number of trades and quoted liquidity, trading volume provides no incremental explanatory power. Thus, the number of trades appears to be a better proxy for information flow. Furthermore, investigation into partitioned volume suggests that the number of trades is more effective than the unexpected volume in explaining volatility. Measures of quoted liquidity also play a significant role in explaining intra day volatility. Bid-ask spreads and quote depth are positively and negatively related to volatility, respectively. Consistent with the lack of information signal, no trade outcomes are negatively related to volatility (JEL G10).

Keywords: volatility, volatility determinants, and market microstructure.

\section{Introduction}

It is widely documented that stock returns display strong conditional heteroscedasticity (Engle and Bollerslev [1986], Schwert[1989]). Stock volatility tends to cluster over time, suggesting volatility contains a predictable component. Though ARCH-type models provide a parsimonious representation of volatility, these models are more

* Financial support from FCAR and SSHRC are gratefully acknowledged. We appreciate the research assistance provided by Pei Shuo Chen.

(Multinational Finance Journal, 1997, vol. 3, no. 3, pp. 199-227)

(C) by Multinational Finance Society, a nonprofit corporation. All rights reserved. DOI: $10.17578 / 1-3-2$ 
statistical than economic in nature. Therefore, an important motivation behind this article is to investigate whether microstructure variables provide an economic grounding for volatility prediction models.

Information flow and market liquidity are two latent variables affecting volatility (Kyle [1985], Glosten and Milgrom [1985], and Admati and Pfleiderer [1988]). Trade activity and quoted liquidity variables are determined jointly by information flow and supply of market liquidity. Thus, the predictable component of volatility is naturally related to microstructure variables such as volume, number of trades, bid-ask spread, and quote depth.

A large number of empirical studies have found a positive contemporaneous relationship between trading volume and volatility (Jain and Joh [1988], Schwert [1989], and Gallant, Rossi, and Tauchen [1992]), so the belief that volume can be used as a proxy for information flow is widely held. For instance, Lamoureux and Lastrapes (1990) find that volume explains the GARCH effect.

However, the role of volume is challenged when other variables such as number of trades and unexpected volume are considered. Jones, Kaul, and Lipson (1994) show that the positive volatility-volume relation disappears when the relation between volatility and number of trades is controlled for, and that trade size has no information content beyond that contained in trade frequency. Bessembinder and Seguin (1992) partition volume into expected and unexpected components, and find that unexpected volume shocks have a relatively larger effect on volatility. Thus, it is necessary to conduct a comprehensive examination of measures of trade activity and re-evaluate the volumevolatility relationship.

Measures of quoted liquidity should also be included in explaining volatility behavior. The quoted bid-ask spread has an adverse selection component (Glosten and Milgrom [1985]). Given the discreteness of quotes, quote sizes are an important decision variable for market makers. Lee, Mucklow, and Ready (1993) show that bid-ask spread and quote depth are two dimensions of market liquidity. Since the electronic system at the Toronto Stock Exchange (TSE) provides traders with easy access to the limit order book, bid-ask spread and quote depth are readily available for assessing market liquidity.

The purpose of this article is to find and interpret the factors that are the source of volatility behavior. The main contributions of this article are as follows. First, intraday trade and quote data are employed to investigate the determinants of intraday volatility, which cannot be 
studied using daily closing prices. Second, different measures of trade activity are used to revisit the volume-volatility relationship, and an extension is made to various volume components. Number of trades, unexpected volume and a non-trading indicator are found to be proxies for information flow. Third, volatility behavior is studied at the individual stock level rather than for a market index. Firm-specific news effects may be neglected in the process of aggregation into the market index used in many previous studies. Fourth, bid-ask spreads and quote depth are used to measure market liquidity. The examination of individual stocks and a traded market index instrument allows for a determination of the impact of quoted liquidity on volatility.

The remainder of this article is organized as follows. Methodological issues dealing with estimating volatility and partitioning volume are discussed in section II. In section III, the data are described and summary statistics are provided. Empirical results and interpretations are given in section IV. A further investigation into volume components is conducted in section V. Concluding remarks are offered in the final section.

\section{Methodology}

\section{A. Estimation of Volatility}

The iterated weighted least squares (IWLS) procedure introduced by Davidian and Carroll (1987) and Schwert (1990) is used to give unbiased estimates of volatility conditional on observable variables. The conditional mean and conditional volatility equations are given by:

$$
R_{j, t}=\sum_{i=1}^{13} \gamma_{j, i} R_{j, t-i}+\sum_{i=1}^{13} \eta_{j, i} \hat{\sigma}_{j, t-i}+\sum_{k=1}^{5} \alpha_{j, k} D_{k, t}+\pi_{j} O P_{t}+u_{j, t}
$$

and

$$
\hat{\sigma}_{j, t}=\sum_{i=1}^{13} \omega_{j, i} \hat{u}_{j, t-i}+\sum_{i=1}^{13} \beta_{j, i} \hat{\sigma}_{j, t-i}+\sum_{k=1}^{5} \mu_{j, k} D_{k, t}+\tau_{j} O P_{t}+v_{j, t}
$$

where $R_{j, t}$ is the return of stock $j$ based on the average of bid and ask quotes at interval $t, \hat{\sigma}_{j, t}$ is the estimated conditional volatility of stock $j$ at time $t, D_{k, t}$ are five dummy variables for days of the week at time $t$, 
$O P_{t}$ is a dummy variable for the opening of the market, and $u_{j, t}$ and $v_{j, t}$ are residual terms. The day-of-the-week dummy variables are used to capture normal differences in means of returns and volatilities by day of the week. Returns for the opening period are treated differently by including the dummy variable $\left(O P_{t}\right)$, because closing stock prices are used to compute returns but are inaccurate reflections of opening values. If the residuals from equation 1 can be approximated by a conditional normal distribution, the estimated conditional volatility is given by

$$
\hat{\sigma}_{j, t}=\left|\hat{u}_{j, t}\right| \sqrt{\pi / 2}
$$

The IWLS procedure requires iterations between the conditional mean equation 1 and the conditional volatility equation 2 : First, equation 1 is estimated without lagged volatility estimates. The residuals are unexpected returns. Second, the residuals generated from equation 1 are transformed by equation 3 to obtain estimates of conditional volatility. $\hat{\sigma}_{j, t}$ is non-negative since it is based on the absolute deviation. Third, equation 2 is estimated by regressing estimates of conditional volatility against lagged unexpected returns, lags of estimated conditional volatility, and day-of-the-week dummy variables. Lagged volatility estimates are included to capture persistence in volatility. Lagged residuals from equation 1 are used to allow for a possible asymmetric relationship between volatility and unexpected returns. Fourth, fitted values from equation 2 are used as regressors in the estimation of equation 1 . Finally, equation 2 is estimated using residuals from the consistent estimation of equation 1. Fitted values from equation 2 are used as estimates of conditional volatility in subsequent regression analyses.

Returns based on bid-ask midpoints instead of transaction-based returns are used to mitigate the bid-ask bounce problem. Kaul and Nimalendran (1990) demonstrate that bid-ask bounce accounts for 50\% of observed volatility. In the absence of trading, public announcements or information revealed in the trading of other related securities may still have an impact on the underlying value. Quote-based returns capture such changes and solve the stale price-problem. However, quote revisions may still be sticky to some extent. Similar to an ARMA model in Stoll and Whaley (1990) to purge the effects of nonsynchronous trading, the autoregressive terms in the conditional mean equation 1 are used to reduce the stickiness problem. 


\section{B. Volume Decomposition}

The Box-Jenkins procedure is used to estimate ARMA models for partitioning volume into expected and unexpected components. Since volume exhibits a consistent daily cycle based on the partial autocorrelations, temporal adjustment at the 13th lag succeeds in removing the temporal component. An examination of the volume series indicates that it can be represented as $\operatorname{ARMA}(3,3)$ with a seasonal adjustment:

$$
V O L_{j, t}=\sum_{i=1}^{3} \phi_{j, i} V O L_{j, t-i}+\sum_{i=0}^{3} \phi_{j, i} V O L_{j, t-13-i}+\varepsilon_{j, t}+\sum_{k=1}^{3} \theta_{j, k} \varepsilon_{j, t-k}
$$

where $V O L_{j, t}$ is trading volume and $\varepsilon_{j, t}$ is the residual volume for stock $j$ for interval $t$. The same model specification with a MA(3) term and a relatively-long autoregressive disturbance term may over-fit the data and decrease efficiency, but this avoids the need to build a model for each stock. Diagnostic checks on the overall acceptability of residual autocorrelations are carried out using the Ljung-Box portmanteau Qstatistic. $\mathrm{Q}$ is approximately $\chi^{2}$ distributed. The null hypothesis that the ARMA disturbances are serially independent is tested. Results show that the $\operatorname{ARMA}(3,3)$ model is adequate to fit the volume series. Residuals and fitted values from equation 4 are used as unexpected volumes and expected volumes, respectively.

\section{Data}

Data are obtained from the time-stamped transaction and quotation files of the Toronto Stock Exchange for the 13-month period from June 1990 to June 1991. These files provide detailed trade and quote information for all companies listed on the TSE. Conversion of quote prices into returns over a fixed time interval is necessary since changes in quotes are not uniformly spaced in time. Each trading day commences at 9:30 a.m. and ends at 4:30 p.m. and is partitioned into 13 half-hour intervals. The total number of intraday observations is 3,536. Since intraday observations are separated by overnight and weekend periods, timeseries data are not uniform in the length of intervals. Rather, at the market opening, information accumulates over a longer period of time, and a call auction usually determines opening prices. Indicator 
variables for open and Monday are used to capture such effects.

The focus on individual stocks instead of an index as in prior research allows for the detection of the relationship between volatility and microstructure variables caused by firm-specific information flow. Since most public or private information is firm specific in nature (Mitchell and Mulherin [1994]), the impact of firm-specific information may be diversified away in the process of aggregation to a market index.

The use of individual stocks is also motivated by the availability of quote data for individual stocks. In this study, component stocks in the Toronto Stock Exchange (TSE) 35 index and the Toronto 35 Index Participation Shares (TIPS) are selected. The TSE 35 index represents the largest and most actively-traded Canadian stocks, so the infrequent trading problem is less serious. Since its inception in March 1990, TIPS has become an actively traded market index instrument. Because it is a traded market index, it has its own bid-ask spread and quote depth, and the results for TIPS have implications for the overall market.

The Toronto Stock Exchange traded stocks in two different ways during the studied time period: on a trading floor and through the Computer Assisted Trading System (CATS). Trading in the more active stocks generally occurs through an integration of the traditional floor trading with an electronic system. Each of these active stocks has a designated market maker that is responsible for maintaining an orderly market. Trading in less active stocks generally takes place through the CATS, which provides access to the Market Order System of Trading (MOST) and to the Limit Order Trading System (LOTS). A few active issues are included in CATS, such as TIPS, and the trend is towards closing floor trading and relying on the electronic system.

The electronic open book system, which was implemented in 1990, allows brokers off the floor to enter orders. Members of the exchange have open access to the book of limit orders as well as to the identity of the brokerage house submitting each limit order. Information on spreads and quote depths is readily available to investors, but a large order trader in the electronic system or CATS has the option of not disclosing that part of an order that is in excess of 5,000 shares. However, liquidity traders may prefer to publicize their orders because the TSE gives priority to disclosed orders over undisclosed orders at the same price.

Prices are adjusted for quarterly dividends, and returns are calculated using the average of bid and ask quotes. Quoted spread is the difference between ask and bid quotes, and the percentage quoted 
spread is calculated using the formula $S P D_{j, t}=\left(A S K_{j, t}-B I D_{j, t}\right) / m_{j, t}$, where $m_{j, t}$ is the bid-ask midpoint at time $t$. Quote depth $\left(Q D_{j, t}\right)$ is the sum of bid and ask sizes. In the results subsequently reported, all of the variables for quoted liquidity are measured at the end of each interval.

The correlations between the estimated volatility and various trade and quote variables for each stock are presented in table 1. As expected, the estimated volatility is positively correlated with volume, expected and unexpected volumes, number of trades, and the quoted spread, and is negatively correlated with quote depth. The augmented Dickey-Fuller tests for presence of unit roots are conducted for volume and number of trades. The null hypothesis of a unit root is rejected for all of these series at the $1 \%$ level. For example, the ADF test statistic on the volume of TIPS has a value of -14.71 , and the MacKinnon critical value at the $1 \%$ level is -3.96 . Results are similar for all of the component stocks.

\section{Empirical Results}

The dynamic relationships between volatility and various trade and quote variables are investigated using the following regression model

$$
\begin{gathered}
\sigma_{j, t}=\alpha_{j}+b_{j, 1} V O L_{j, t}+b_{j, 2} \operatorname{VOLR}_{j, t}+b_{j, 3} N T_{j, t} \\
+b_{j, 4} D U M_{j, t}+b_{j, 5} S P D_{j, t-1}+b_{j, 6} Q D_{j, t-1} \\
+b_{j, 7} O P_{t}+b_{j, 8} M O N_{t}+\sum_{i=1}^{13} c_{j, i} \sigma_{j, t-i}+e_{j, t},
\end{gathered}
$$

where $V O L_{j, t}$ is trading volume for interval $t, V O L R_{j, t}$ is trading volume when the trades during the interval are classified as a seller-initiated order (i.e., $V O L R_{j, t}$ equals $V O L_{j, t}$ if the closing transaction price for interval $t$ is lower than the average of bid and ask quotes at the end of interval $t-1$,otherwise $V O L R_{j, t}$ is zero), $N T_{j, t}$ is number of trades for interval $t, D U M_{j, t}$ is a dummy variable which has a value of one for nontrading outcome at time $t$ and zero otherwise, $S P D_{j, t}$ is the percent quoted spread, and $Q D_{j, t}$ is quote depth at the end of interval $t$. Dummy variables for the opening $\left(O P_{t}\right)$ and Monday $\left(M O N_{t}\right)$ are included to control for temporal regularities of the volatility series. The lagged 
TABLE 1. Correlations of Volatility Estimates of Firms' Stock Returns with the Microstructure Variables

\begin{tabular}{|c|c|c|c|c|c|c|}
\hline \multirow[b]{2}{*}{ Variables } & \multicolumn{6}{|c|}{ Tick } \\
\hline & $\mathrm{AL}$ & B & BMO & BNS & BVI & CAE \\
\hline$V O L_{t}$ & .3361 & .2499 & .0876 & .2917 & .3020 & .1783 \\
\hline$E V O L_{t}$ & .2351 & .2762 & .2544 & .2556 & .2620 & .2556 \\
\hline$U V O L_{t}$ & .1216 & .2031 & .0780 & .2284 & .2699 & 1480 \\
\hline$N T_{t}$ & .2501 & .3522 & .3461 & .2673 & .4070 & .5140 \\
\hline$S P D_{t-1}$ & .0078 & .1215 & .1750 & .1340 & .1186 & .1453 \\
\hline \multirow[t]{2}{*}{$Q D_{t-1}$} & -.0069 & -.1732 & -.1329 & -.1817 & -.0397 & -.1032 \\
\hline & \multicolumn{6}{|c|}{ Tick } \\
\hline Variables & $\mathrm{CM}$ & $\mathrm{CP}$ & CTR.A & ECO & GOU & IMO.A \\
\hline$V O L_{t}$ & .2464 & .1660 & .1643 & .3293 & .1244 & .3097 \\
\hline$E V O L_{t}$ & .2867 & .2793 & .3782 & .3830 & .2117 & .3063 \\
\hline$U V O L_{t}$ & .1934 & .1404 & .0095 & .2100 & .1021 & 2468 \\
\hline$N T_{t}$ & .3507 & .4091 & .1286 & .4458 & .3829 & .4696 \\
\hline$S P D_{t-1}$ & .2698 & .0979 & .0411 & .0830 & .1756 & . 1926 \\
\hline \multirow[t]{2}{*}{$Q D_{t-1}$} & -.1468 & -.1500 & -.0087 & -.0591 & -.0567 & -.0610 \\
\hline & \multicolumn{6}{|c|}{ Tick } \\
\hline Variables & IMS & LAC & LDM.B & $\mathrm{MB}$ & MCL & $\mathrm{N}$ \\
\hline$V O L_{t}$ & .1912 & .3482 & .2455 & .2681 & .1818 & .0615 \\
\hline$E V O L_{t}$ & .2261 & .3239 & .2471 & .2542 & .1851 & 2699 \\
\hline$U V O L_{t}$ & .1582 & .2729 & .2027 & .2214 & .1478 & .0543 \\
\hline$N T_{t}$ & .3587 & .4686 & .4401 & .3589 & .3909 & .4451 \\
\hline$S P D_{t-1}$ & .2336 & .0834 & .0654 & .1118 & .1507 & .0811 \\
\hline \multirow[t]{2}{*}{$Q D_{t-1}$} & -.1163 & -.0904 & -.0668 & -.1408 & -.1655 & -.1233 \\
\hline & \multicolumn{6}{|c|}{ Tick } \\
\hline Variables & NA & NOR & NTL & NVA & PDG & POW \\
\hline$V O L_{t}$ & .2397 & .2274 & .3408 & .2534 & .4046 & 2593 \\
\hline$E V O L_{t}$ & .1255 & .2614 & .2580 & .1546 & .3722 & .2263 \\
\hline$U V O L_{t}$ & .2125 & .1794 & .2750 & .2060 & .2949 & .2232 \\
\hline$N T_{t}$ & .2732 & .4038 & .4378 & .2587 & .4770 & .2594 \\
\hline$S P D_{t-1}$ & .1551 & .1172 & 1360 & .1594 & .0439 & .2370 \\
\hline \multirow[t]{2}{*}{$Q D_{t-1}^{t-1}$} & -.1365 & -.0963 & -.0826 & -.2232 & -.1034 & -.0932 \\
\hline & \multicolumn{6}{|c|}{ Tick } \\
\hline Variables & RGO & RY & SCC & STE.A & STM & TAU \\
\hline$V O L_{t}$ & .1794 & .0960 & .1782 & .2160 & .1754 & .2327 \\
\hline$E V O L_{t}$ & .0232 & .2795 & .1191 & .2773 & .1899 & 1892 \\
\hline$U V O L_{t}$ & .2042 & .0726 & .1693 & .1723 & .1518 & 2075 \\
\hline$N T_{t}$ & .3686 & .3746 & . 4409 & .4519 & .4236 & .1274 \\
\hline$S P D_{t-1}$ & .1010 & .1323 & .1887 & .2707 & .2155 & .1961 \\
\hline$Q D_{t-1}$ & -.0869 & -.1138 & -.1485 & -.1001 & -.1376 & -.1376 \\
\hline
\end{tabular}

(Continued) 
TABLE 1. (Continued)

\begin{tabular}{lcccccc}
\hline & \multicolumn{7}{c}{ Tick } \\
\cline { 2 - 7 } Variables & TD & TEK.B & TOC & TRP & VO & TIPS \\
\hline VOL $_{t}$ & .1718 & .2748 & .2710 & .3452 & .1977 & .1781 \\
VVOL $_{t}$ & .1981 & .2110 & .2210 & .1066 & .2002 & .2433 \\
UVOL & .1307 & .2365 & .2299 & .3297 & .1711 & .1514 \\
$N T_{t}$ & .3480 & .4297 & .4151 & .2528 & .4044 & .2387 \\
$S P D_{t-1}$ & .1085 & .1682 & .1611 & .1505 & .2575 & .1350 \\
$Q D_{t-1}$ & -.1784 & -.1083 & -.1313 & -.1761 & -.0721 & -.1178 \\
\hline
\end{tabular}

Note: The correlations of the volatility estimate with each of the six microstructure variables for each stock in the TSE 35 Index and TIPS are presented in this table. Tick refers to the ticker symbols for the companies included in the TSE 35 Index (see the appendix for the corresponding company names). The volatility estimates are obtained using the iterated weighted-least squares (IWLS) procedure as in Schwert (1990). The six microstructure variables are: $V O L_{t}$ or volume; $E V O L_{t}$ or expected volume; $U V O L_{t}$ or unexpected volume; $N T_{t}$ or number of trades; $S P D_{t-1}$ or the percent quoted spread; and $Q D_{t-1}$ or quote depth (defined as the sum of the bid and ask depths).

volatilities are added to account for volatility clustering over time and to remove autocorrelation in residuals. The coefficient for $O P_{t}$ is found to be significant for most of the 35 stocks. The objectives of the above regression equation are to re-evaluate the role of trading volume, to assess the relative importance of number of trades, and to examine the impact of quoted liquidity. Robust estimates for the standard errors of coefficients are computed using White's (1980) method. ${ }^{2}$

\section{A. Trading Volume}

Surveying the literature, Karpoff (1987) reports that trading volume is positively related to volatility. However, it is not completely resolved whether such a positive volume-volatility relationship holds with the inclusion of other measures of trade activity and quoted liquidity. Table 2 presents estimates for regression 5 which allow for a test of whether trading volume is an effective proxy for information flow. Based on table 2, the null hypothesis of no relationship between volume and volatility is not overwhelmingly rejected. Coefficients for volume are statistically significant and positive for only 15 of the 35 stocks at the $1 \%$ level. Thus, trading volume appears to have no additional explanatory power when volatility is conditioned on number of trades and measures of quoted liquidity.

2. Regressions of volatility on simple combinations of trade or quote variables are performed, but results are not reported in order to save valuable journal space. 
TABLE 2. Results of Regressions of Estimated Volatility on Various Trade and Quote Variables Based on Equation 5

\begin{tabular}{|c|c|c|c|c|c|c|}
\hline \multirow[b]{2}{*}{ Variables } & \multicolumn{6}{|c|}{ Tick } \\
\hline & $\mathrm{AL}$ & B & BMO & BNS & BVI & CAE \\
\hline \multirow[t]{2}{*}{$V O L_{j, t}$} & .1366 & .0382 & .0039 & .1815 & .4967 & .0178 \\
\hline & $(2.98)^{* *}$ & (1.81) & (1.42) & $(3.87)^{* *}$ & $(5.27)^{* *}$ & $(.53)$ \\
\hline \multirow{2}{*}{$V O L R_{j, t}$} & -.0117 & .0955 & .1183 & -.0712 & -.3479 & .0107 \\
\hline & $(-.25)$ & $(3.01)^{* *}$ & $(4.52)^{* *}$ & $(-1.01)$ & $(-2.17)^{*}$ & $(.14)$ \\
\hline \multirow[t]{2}{*}{$N T_{j, t}$} & .8077 & .2165 & .5932 & .2839 & 3.1248 & 5.7240 \\
\hline & $(8.25)^{* *}$ & $(7.89)^{* *}$ & $(9.60) * *$ & $(4.68)^{* *}$ & $(5.62)^{* *}$ & $(9.75)^{* *}$ \\
\hline \multirow[t]{2}{*}{$D U M_{j, t}$} & -.1234 & -.0002 & -.0262 & -.0878 & -.0624 & .0069 \\
\hline & $(-8.70)^{* *}$ & $(-.00)$ & $(-2.19)^{*}$ & $(-3.16)^{* *}$ & $(-3.47)^{* *}$ & $(.22)$ \\
\hline \multirow[t]{2}{*}{$S P D_{j, t-1}$} & .2373 & .2981 & .3292 & .3399 & .0998 & .1647 \\
\hline & $(7.61)^{* *}$ & $(9.10)^{* *}$ & $(10.93)^{* *}$ & $(9.95)^{* *}$ & $(7.21)^{* *}$ & $(6.18)^{* *}$ \\
\hline \multirow[t]{2}{*}{$Q D_{j, t-1}$} & -1.8247 & -.7809 & -1.0724 & -1.5787 & -1.8911 & -2.3218 \\
\hline & $(-5.79) * *$ & $(-6.39)^{* *}$ & $(-5.38)^{* *}$ & $(-8.29)^{* *}$ & $(-3.79)^{* *}$ & $(-6.62)^{* *}$ \\
\hline \multirow[t]{2}{*}{$O P_{t}$} & .2816 & .1440 & .2239 & .4678 & .2003 & .1114 \\
\hline & $(6.09)^{* * *}$ & $(5.58)^{* *}$ & $(7.51)^{* *}$ & $(8.53)^{* *}$ & $(6.02)^{* *}$ & $(1.45)$ \\
\hline \multirow[t]{2}{*}{$M O N_{t}$} & .2194 & .0302 & -.0683 & .1132 & .2355 & .6572 \\
\hline & $(1.27)$ & $(.34)$ & $(-.68)$ & $(.58)$ & (1.82) & $(2.20)^{*}$ \\
\hline$\sigma_{j, t-i}$ & {$[2.43]^{*}$} & {$[4.32]^{* *}$} & {$[6.23]^{* *}$} & {$[4.12]^{* *}$} & {$[8.23]^{* *}$} & {$[9.87]^{* *}$} \\
\hline DW & 1.981 & 1.999 & 2.038 & 2.003 & 2.009 & 1.935 \\
\hline $\mathrm{R}^{2}$ & .2281 & .2243 & .2534 & .2262 & .2662 & .3153 \\
\hline
\end{tabular}

(Continued) 
TABLE 2. (Continued)

\begin{tabular}{|c|c|c|c|c|c|c|}
\hline Variables & $\mathrm{CM}$ & $\mathrm{CP}$ & CTR.A & ECO & GOU & IMO.A \\
\hline \multirow[t]{2}{*}{$V O L_{j, t}$} & .0942 & .1125 & .0435 & .3738 & .1415 & .2277 \\
\hline & $(3.49)^{* *}$ & $(4.18)^{* *}$ & (.99) & $(2.20)^{*}$ & (1.07) & $(2.35)^{*}$ \\
\hline \multirow{2}{*}{$V O L R_{j, t}$} & .0532 & -.1009 & .0970 & -.1754 & -.1002 & .0438 \\
\hline & (1.11) & $(-3.75)^{* *}$ & (1.36) & $(-.83)$ & $(-.70)$ & (.39) \\
\hline \multirow{2}{*}{$N T_{j, t}$} & .9295 & .6730 & 1.2949 & 3.6403 & 3.8687 & 1.3823 \\
\hline & $(8.68)^{* *}$ & $(10.50)^{* *}$ & $(9.37)^{* *}$ & $(6.38)^{* *}$ & $(7.22)^{* *}$ & $(6.38)^{* *}$ \\
\hline \multirow{2}{*}{$D U M_{j, t}$} & -.0430 & -.0402 & -.0505 & -.1481 & -.0917 & -0.0108 \\
\hline & $(-3.75)^{* *}$ & $(-1.11)$ & $(-4.49) * *$ & $(-5.31)^{* *}$ & $(-3.79)^{* *}$ & $(-.87)$ \\
\hline \multirow[t]{2}{*}{$S P D_{j, t-1}$} & .2600 & .3113 & .1581 & .2024 & .1451 & .1568 \\
\hline & $(9.12)^{* *}$ & $(10.67)^{* *}$ & $(7.68)^{* *}$ & $(6.83) * *$ & $(8.45)^{* *}$ & $(5.88)^{* *}$ \\
\hline \multirow[t]{2}{*}{$Q D_{j, t-1}$} & -1.4648 & -1.0943 & -2.5635 & -3.1073 & -3.2870 & -3.2712 \\
\hline & $(-5.16) * *$ & $(-5.62) * *$ & $(-6.78) * *$ & $(-3.65)^{* *}$ & $(-5.55)^{* *}$ & $(-5.38)^{* *}$ \\
\hline \multirow[t]{2}{*}{$O P_{t}$} & .3442 & .1924 & .1557 & .9253 & .3233 & .2188 \\
\hline & $(7.59)^{* *}$ & $(5.05)^{* *}$ & $(4.78)^{* *}$ & $(8.94) * *$ & $(6.95)^{* *}$ & $(8.21)^{* *}$ \\
\hline \multirow[t]{3}{*}{$M O N_{t}$} & .9265 & -.0175 & -.1284 & .6738 & .2311 & .1323 \\
\hline & $(.62)$ & $(-.12)$ & $(-1.01)$ & $(2.09)^{*}$ & (1.14) & (1.46) \\
\hline & {$[5.54]^{* *}$} & {$[3.46]^{* *}$} & {$[5.97]^{* *}$} & {$[3.75]^{* *}$} & {$[6.34]^{* *}$} & {$[3.62]^{* *}$} \\
\hline$D W$ & 2.000 & 1.975 & 1.988 & 1.985 & 2.009 & 1.989 \\
\hline$R^{2}$ & .2626 & .2337 & .1764 & .2968 & .2334 & .3060 \\
\hline
\end{tabular}


TABLE 2. (Continued)

\begin{tabular}{|c|c|c|c|c|c|c|}
\hline \multirow[b]{2}{*}{ Variables } & \multicolumn{5}{|c|}{ Tick } & \multirow[b]{2}{*}{$\mathrm{N}$} \\
\hline & IMS & LAC & LDM.B & MB & MCL & \\
\hline \multirow{2}{*}{$V O L_{j, t}$} & .0905 & .2149 & .0630 & .2207 & .0134 & -.0041 \\
\hline & (1.60) & $(3.25)^{* *}$ & (1.03) & $(2.41)^{*}$ & $(.32)$ & $(-.38)$ \\
\hline \multirow{2}{*}{$V O L R_{j, t}$} & -.0177 & -.0396 & -.0267 & .0089 & .0773 & .0045 \\
\hline & $(-.25)$ & $(-.46)$ & $(-.37)$ & $(.08)$ & (1.42) & $(.42)$ \\
\hline \multirow[t]{2}{*}{$N T_{j, t}$} & 1.8741 & 2.4102 & .7378 & 1.4072 & 1.8854 & 1.2768 \\
\hline & $(9.01)^{* *}$ & $(9.65)^{* *}$ & $(2.59)^{* *}$ & $(8.45)^{* *}$ & $(10.24)^{* *}$ & $(9.96)^{* *}$ \\
\hline \multirow[t]{2}{*}{$D U M_{j, t}$} & -.0499 & -.1090 & -.1388 & -.0493 & -.0572 & -.0535 \\
\hline & $(-5.02) * *$ & $(-4.57) * *$ & $(-2.85)^{* *}$ & $(-2.83) * *$ & $(-4.96)^{* *}$ & $(-3.93) * *$ \\
\hline \multirow[t]{2}{*}{$S P D_{j, t-1}$} & .1437 & .2258 & .3449 & .1815 & .2003 & .2257 \\
\hline & $(5.95)^{* *}$ & $(8.39) * *$ & $(4.19)^{* *}$ & $(8.98)^{* *}$ & $(7.23)^{* *}$ & $(7.41)^{* *}$ \\
\hline \multirow[t]{2}{*}{$Q D_{j, t-1}$} & -2.0491 & -2.7404 & -2.2354 & -2.3671 & -2.7827 & -2.8920 \\
\hline & $(-4.17)^{* *}$ & $(-5.83)^{* *}$ & $(-3.55) * *$ & $(-7.25) * *$ & $(-7.30)^{* *}$ & $(-7.50) * *$ \\
\hline \multirow[t]{2}{*}{$O P_{t}$} & .2026 & .6982 & .5665 & .2028 & .2058 & .3758 \\
\hline & $(6.26)^{* *}$ & $(9.10)^{* *}$ & $(8.24)^{* *}$ & $(5.51)^{* *}$ & $(6.05)^{* *}$ & $(8.26)^{* * *}$ \\
\hline \multirow[t]{2}{*}{$M O N_{t}$} & .0546 & .3258 & .2076 & -.1054 & .0984 & -.0124 \\
\hline & $(.50)$ & $(1.11)$ & $(.92)$ & $(-.76)$ & $(.79)$ & $(-.10)$ \\
\hline$\sigma_{j, t-i}$ & {$[12.03]^{* *}$} & {$[7.08] * *$} & {$[5.16] * *$} & {$[7.74] * *$} & {$[4.21]^{* *}$} & {$[3.84]^{* *}$} \\
\hline$D W$ & 1.997 & 1.954 & 1.890 & 2.032 & 1.986 & 1.975 \\
\hline$R^{2}$ & .2248 & .3138 & .2704 & .2280 & .2295 & .2996 \\
\hline
\end{tabular}

(Continued) 
TABLE 2. (Continued) 
TABLE 2. (Continued)

\begin{tabular}{|c|c|c|c|c|c|c|}
\hline \multirow[b]{2}{*}{ Variables } & \multicolumn{6}{|c|}{ Tick } \\
\hline & RGO & RY & $\mathrm{SCC}$ & STE.A & STM & TAU \\
\hline \multirow[t]{2}{*}{$V O L_{j, t}$} & .0122 & .0635 & .0847 & .1970 & .2181 & .3046 \\
\hline & $(.95)$ & $(2.02)^{*}$ & (1.49) & $(1.73)$ & $(1.80)$ & $(6.34) * *$ \\
\hline \multirow[t]{2}{*}{$V O L R_{j, t}$} & .0295 & -.0611 & .1035 & .4530 & -.1644 & -.1512 \\
\hline & $(2.34)^{*}$ & $(-1.89)$ & $(.72)$ & $(1.77)$ & $(-.79)$ & $(-2.64) * *$ \\
\hline \multirow{2}{*}{$N T_{j, t}$} & 2.4283 & .8894 & 9.4841 & 4.5800 & 5.7193 & .2786 \\
\hline & $(6.27)^{* *}$ & $(9.68)^{* *}$ & $(6.55)^{* *}$ & $(7.65)^{* *}$ & $(6.21)^{* *}$ & $(4.63)^{* *}$ \\
\hline \multirow[t]{2}{*}{$D U M_{j, t}$} & -.1140 & -.0424 & -.0298 & -.0298 & -.0358 & -.0401 \\
\hline & $(-4.50)^{* *}$ & $(-2.79) * *$ & $(-.81)$ & $(-.87)$ & $(-1.40)$ & $(-3.02) * *$ \\
\hline \multirow[t]{2}{*}{$S P D_{j, t-1}$} & .2325 & .2748 & .0580 & .1713 & .1247 & .2637 \\
\hline & $(10.50)^{* *}$ & $(8.93)^{* *}$ & $(4.70)^{* *}$ & $(6.83)^{* *}$ & $(6.62)^{* *}$ & $(11.91)^{* *}$ \\
\hline \multirow[t]{2}{*}{$Q D_{j, t-1}$} & -1.0658 & -1.2711 & -1.9182 & -5.3048 & -2.5246 & -.8322 \\
\hline & $(-4.36)^{* *}$ & $(-5.18)^{* *}$ & $(-4.94) * *$ & $(-4.93) * *$ & $(-3.44)^{* *}$ & $(-5.17)^{* *}$ \\
\hline \multirow[t]{2}{*}{$O P_{t}$} & .3230 & .2643 & .2102 & .3715 & .2239 & .1811 \\
\hline & $(5.54) * *$ & $(8.06)^{* *}$ & $(4.39) * *$ & $(5.17)^{* *}$ & $(6.01)^{* *}$ & $(5.74) * *$ \\
\hline \multirow[t]{2}{*}{$M O N_{t}$} & -.2286 & -.5845 & -.1042 & -.3018 & -.0786 & .0406 \\
\hline & $(-.99)$ & $(-.05)$ & $(-.55)$ & $(-.98)$ & $(-.50)$ & $(.30)$ \\
\hline$\sigma_{j, t-i}$ & {$[7.67]^{* *}$} & {$[5.83]^{* *}$} & {$[7.15]^{* *}$} & {$[5.96]^{* *}$} & {$[4.21]^{* *}$} & {$[16.97]^{* *}$} \\
\hline$D W$ & 2.007 & 2.033 & 2.020 & 1.993 & 2.003 & 2.020 \\
\hline$R^{2}$ & .2184 & .2296 & .2497 & .2824 & .2412 & .1945 \\
\hline
\end{tabular}

(Continued) 
TABLE 2. (Continued)

\begin{tabular}{|c|c|c|c|c|c|c|}
\hline \multirow[b]{2}{*}{ Variables } & \\
\hline & TD & TEK.B & TOC & TRP & VO & TIPS \\
\hline$V O L_{j, t}$ & $\begin{array}{l}.0698 \\
(1.66)\end{array}$ & $\begin{array}{c}.1550 \\
(2.80)^{* *}\end{array}$ & $\begin{array}{c}.1257 \\
(3.72)^{* *}\end{array}$ & $\begin{array}{l}.0819 \\
(1.51)\end{array}$ & $\begin{array}{l}.1601 \\
(1.72)\end{array}$ & $\begin{array}{l}.4302 \\
(1.34)\end{array}$ \\
\hline$V O L R_{j, t}$ & $\begin{array}{c}-.0978 \\
(-1.94)\end{array}$ & $\begin{array}{l}-.0280 \\
(-.34)\end{array}$ & $\begin{array}{l}-.0492 \\
(-.94)\end{array}$ & $\begin{array}{l}.2883 \\
(1.46)\end{array}$ & $\begin{array}{l}-.1300 \\
(-1.28)\end{array}$ & $\begin{array}{l}-.1343 \\
(-.34)\end{array}$ \\
\hline$N T_{j, t}$ & $\begin{array}{r}.7396 \\
(6.37) * *\end{array}$ & $\begin{array}{l}2.6818 \\
(5.64) * *\end{array}$ & $\begin{array}{c}2.7967 \\
(13.52) * *\end{array}$ & $(3.31)^{* *}$ & $\begin{array}{c}1.7231 \\
(10.68) * *\end{array}$ & $\begin{array}{r}.8138 \\
(2.41)^{*}\end{array}$ \\
\hline$D U M_{j, t}$ & $\begin{array}{c}-.0714 \\
(-3.49)^{* *}\end{array}$ & $\begin{array}{c}-.0955 \\
(-4.70)^{* *}\end{array}$ & $\begin{array}{c}.0234 \\
(2.11)^{*}\end{array}$ & $\begin{array}{c}-.0410 \\
(-3.21) * *\end{array}$ & $\begin{array}{c}-.0291 \\
(-3.77)^{* *}\end{array}$ & $\begin{array}{c}-.6157 \\
(-4.32) * *\end{array}$ \\
\hline$S P D_{j, t-1}$ & .2808 & .1287 & .2414 & $\begin{array}{c}.3011 \\
(996) * *\end{array}$ & $\begin{array}{c}.1898 \\
526) * *\end{array}$ & .3058 \\
\hline$Q D_{j, t-1}$ & -1.8070 & -2.2786 & -.6827 & -1.2028 & -6.1519 & -.4492 \\
\hline$O P_{t}$ & $\begin{array}{c}.3791 \\
(7.77)^{* *}\end{array}$ & $\begin{array}{c}.3067 \\
(8.30)^{* *}\end{array}$ & $\begin{array}{c}.1500 \\
(4.45)^{* *}\end{array}$ & $\begin{array}{c}.1491 \\
(4.99) * *\end{array}$ & $\begin{array}{c}.1644 \\
(6.67)^{* *}\end{array}$ & $(8.01)^{* *}$ \\
\hline$M O N_{t}$ & $\begin{array}{l}.0196 \\
(.12)\end{array}$ & $\begin{array}{l}.0225 \\
(-.14)\end{array}$ & $\begin{array}{l}-.1204 \\
(-.89)\end{array}$ & $\begin{array}{c}-.3477 \\
(-3.05)^{* *}\end{array}$ & $\begin{array}{l}.0093 \\
(.10)\end{array}$ & $\begin{array}{l}.0100 \\
(.80)\end{array}$ \\
\hline$\sigma_{j, t-i}$ & {$[2.81]^{*}$} & {$[3.64]^{* *}$} & {$[9.11]^{* *}$} & {$[3.01]^{*}$} & {$[6.04]^{* *}$} & {$[11.91]^{* *}$} \\
\hline $\begin{array}{l}\text { DW } \\
\mathrm{R}^{2}\end{array}$ & $\begin{array}{c}2.020 \\
.2139\end{array}$ & $\begin{array}{l}2.016 \\
.2687\end{array}$ & $\begin{array}{l}1.999 \\
.2493\end{array}$ & $\begin{array}{c}1.916 \\
.2618\end{array}$ & $\begin{array}{l}1.966 \\
.2330\end{array}$ & $\begin{array}{l}2.017 \\
.1717\end{array}$ \\
\hline
\end{tabular}

Note: Regression of estimated volatility $\sigma_{i, j}$ on trade and quote variables is given by $\sigma_{j, t}=a_{j}+b_{j, 1} V O L_{j, t}+b_{j, 2} V O L R_{j, t}+b_{j, 3} N T_{j, t}+b_{j, 4} D U M_{j, t}+$ $b_{j, 5} S P D_{j, t-1}+b_{j, 6} Q D_{j, t, l}+b_{j, 7} O p t+b_{j, 8} M O N_{t}+\sum_{i, j} c_{j, i} \sigma_{j, t-i}+e_{i, p}$ where $V O L_{j, t}$ is volume of stock $j$ at time $t, V O L R_{j, t}$ is volume when the trades during the interval are classified as a sell order (i.e. $V O L R_{j, t}=V O L L_{j, t}$ if $p_{j, t}<m_{j, t-1}$, where $p_{j, t}$ is price at time $t$ and $m_{j, t-1}$ is the average of bid and ask quotes at time $\mathrm{t}-1$; otherwise $\left.V O L R_{j, t}=0\right), N T_{j, t}$ is number of trades, $D U M_{j, t}$ is the indicator variable for no trade outcomes, $S P D_{j, t 1}$ is the percent quoted spread; $Q D_{j, t-1}$ The dependent variable is estimated volatility based on the IWLS procedure used by Schwert (1990). Durbin-Watson (DW) statistics and adjusted R are provided. $*(* *)$ denotes statistical significance at the 5\% (1\%) level. Test statistics for individual coefficients are t-statistics (in parentheses) computed using White (1980) standard errors. Test statistics for lagged volatility coefficients are F-statistics (in brackets) for the hypothesis that the sum of the 13 coefficients is zero. 
The lack of significance for trading volume can be explained by the net effect of the two competing influences of information flow and supply of market liquidity. On the one hand, Clark (1973), Tauchen and Pitts (1983), and Lamoureux and Lastrapes (1990) show that volume and volatility are jointly endogenous variables which move together in response to information shocks. On the other hand, a portion of trading volume is due to expected liquidity trading. Easley and O'Hara (1992) define it as "normal" volume. The greater the normal volume, the lower the volatility since informed traders effectively can hide their identity.

Moreover, volume of liquidity trading is affected by transaction costs and is negatively related to the degree of asymmetric information. Our results for trading volume are consistent with a model of transaction-cost-elastic liquidity trading by George, Kaul, and Nimalendran (1994). They show that the common belief that volume is positively related to asymmetric information may not hold when transaction costs are considered. Their model implies that the relationship between volume and informational asymmetry (or volatility) is ambiguous.

Whether the volume-volatility relationship is symmetric to changes in stock price is also investigated. The indicator variable, $V O L R_{j, t}$, differentiates the case of buyer-initiated volume from that of sellerinitiated volume. Its coefficient captures the interaction of volume and changes in stock price. The estimated coefficients for $V O L R_{j, t}$ are significant and positive for only 4 of the 35 stocks. Therefore, the direction of trading volume appears to have no impact on volatility. This can be explained by the following reasons. On the one hand, buyer-initiated volume may have stronger information. The choice of a particular stock to purchase often conveys favorable firm-specific information, but institutional investors have many liquidity reasons to dispose of a stock (Chan and Lakonishok [1993]). On the other hand, it is conceivable that an inverse relationship between volatility and changes in stock prices due to a leverage effect leads to a greater impact on volatility from the seller-initiated volume. The observed symmetric volume-volatility relationship may be the net effect of these competing factors.

\section{B. Trading Frequency}

Whether number of trades has a positive impact on volatility is investigated next. Based on table 2, the null hypothesis of no 
relationship between number of trades and volatility is strongly rejected. The coefficients for $N T_{j, t}$ are significantly positive for all 35 stocks at the $1 \%$ level (and at the 5\% level for TIPS). The positive relationship between number of trades and volatility is not sensitive to whether volume is included.

Differences in the predictions on the volatility impact of trade frequency are attributed largely to the underlying assumption on the role of trade size. A negative impact from trade frequency may result when trade size is viewed as an indicator variable of information flow (Easley and O'Hara [1987]). Madhavan (1992) suggests that trading frequency, given trading volumes, may be negatively related to spreads, and his conclusion extends to volatility. However, trade frequency may have a positive relationship with volatility if it reflects the rate of information flow. This is based on the Mixture of Distribution Hypothesis that trades take place at a uniform rate in transaction time (Harris [1987]). Jones et al. (1994) show that trade size on average has no incremental information content beyond that contained in number of trades. Their finding can be viewed as a direct test of the hypothesis, and it suggests that number of trades is the mixing variable.

Our findings strongly support that number of trades is associated with the rate of information flow. This is consistent with the finding of Jones et al. (1994) that volatility is primarily determined by number of trades rather than trading volume. The empirical evidence that information content is not monotonically related to trade size helps to explain our results. For example, in their study of tender-offer targets, Barclay and Warner (1993) find that medium-size orders are more likely to be used by informed traders.

A non-trading outcome is a special case of trading frequency. Whether volatility is reduced over time if non-trading outcomes are observed is examined. The null hypothesis of no relationship between non-trading outcomes and volatility is rejected across most stocks. Based on table 2, coefficients of $D U M_{j, t}$ are statistically negative for 24 of the 35 stocks at the $1 \%$ level. The negative sign is consistent with the prediction of Easley and O'Hara (1992) that a non-trading outcome serves as a signal for a lack of information arrival to the market. The coefficient of $D U M_{j, t}$ for TIPS is significantly negative at the $1 \%$ level, suggesting that the negative relationship is likely to be market-wide.

\section{Quoted Liquidity}

A market maker faces an adverse selection problem when trading with 
both informed traders and liquidity traders (Glosten and Milgrom [1985], Madhavan [1992]). Bid-ask spreads allow the market maker to recover losses to informed traders from profits from liquidity traders. Thus, the quoted spread is inversely related to the supply of market liquidity and positively related to information flow. The null hypothesis of no relation between bid-ask spreads and volatility is rejected for 29 of the 35 stocks and for TIPS at the $1 \%$ level, based on table 2. The estimated coefficients for $S P D_{j, t-1}$ are positive for most of the 35 stocks. This suggests that a significant portion of quoted spread is due to information flow.

The expectation is that quote depths are negatively related to volatility. Lee, Mucklow, and Ready (1993) show that quote depth is another dimension of market liquidity, and find that spreads and quote depth are two negatively related measures of market liquidity. A publicized limit order has a similar effect to the "sunshine" trading strategy that liquidity traders signal their motives for trading. Thus, quote depth is a positive indicator variable for market liquidity.

Based on table 2, the estimated coefficients for $Q D_{j, t-1}$ are statistically significant and negative for all 35 stocks and TIPS at the 1\% level. The negative sign for quoted depth is consistent with the belief that most limit orders are used by non-informed traders. Since limit orders can be "picked off" by other investors, limit order submitters provide the market with a free trading option whose value depends on the short-run volatility of prices. Quote depths play a more significant role as discreteness problems get more severe. When the minimum trading tick is relatively large (as for low-priced stocks), the market maker and submitters of limit orders line up to supply liquidity to the market. The market maker may choose not to compete with other liquidity suppliers, given an increasing possibility of information arrival. This is reflected in a reduced quote depth. Thus, shifts in market liquidity may be more easily detected in quote depths than in spreads.

The two measures of market liquidity are not perfect since information on quoted liquidity is not limited to the bid-ask spread and quote depth associated with the best quote prices. The electronic system adopted at the TSE publicly discloses the next levels of bids and asks with their associated sizes and the brokerage house identity on each limit order. Reputations can be developed for providing superior liquidity and thus become an additional factor. 


\section{Further Investigation of Trading Volume}

Both information flow and market liquidity supply appear to determine trading volume. The role of trading volume is further investigated by partitioning volume into its expected and unexpected components. Since abnormal volumes are more likely to be associated with the arrival of new information, the expectation is that unexpected volume is positively related to volatility. The following regression includes both expected and unexpected volumes as independent variables, in addition to other trade activity and quote liquidity variables. Bessembinder and Seguin (1993) run a similar regression on futures. The regression is

$$
\begin{aligned}
\sigma_{j, t} & =\alpha_{j}+b_{j, 1} \mathrm{EVOL}_{j, t}+b_{j, 2} \mathrm{UVOL}_{j, t}+b_{j, 3} N T_{j, t}+b_{j, 4} \mathrm{DUM}_{j, t} \\
& +b_{j, 5} S P D_{j, t-1}+b_{j, 6} Q D_{j, t-1}+b_{j, 7} O P_{t}+\sum_{i=1}^{13} c_{j, i} \sigma_{j, t-i}+e_{j, t}
\end{aligned}
$$

where expected volume, $E V O L_{j, t}$, and unexpected volume, $U V O L_{j, t}$, are fitted values and residuals generated by the $\operatorname{ARMA}(3,3)$ model for interval $t$, and all the other variables are as defined previously. Since expected and unexpected volumes are generated from first-stage ARMA models, generated-regressor problems (Pagan [1984]) may be relevant. Because t-statistics in the second-stage regression tend to be overstated for the estimated coefficients of expected volume, the results may be inconclusive for those stocks with significant expected volume. When both expected and unexpected volumes are used, the t-statistics of the OLS estimates for expected volume are overstated due to the incorrect estimation of standard errors. Thus, the results of the regressions that involve both expected and unexpected volumes are interpreted herein with caution.

Results for regression 6 are reported in table 3. Coefficients for unexpected volume are statistically significant and positive for 22 of the 35 stocks at the $1 \%$ level. In contrast, the coefficients for expected volume are significant for only 7 of the 35 stocks. This is consistent with the finding of Bessembinder and Seguin (1993) that unexpected volume shocks have a larger impact on volatility. However, the relationship between volatility and number of trades is even stronger; the coefficients for $N T_{j, t}$ are significant and positive for 35 stocks at the $1 \%$ level and for TIPS at the 5\% level. The adjusted R-squares are 
TABLE 3. Results of Regressions of Estimated Volatility on Various Trade and Quote Variables Based on Equation 6

\begin{tabular}{|c|c|c|c|c|c|c|}
\hline \multirow[b]{2}{*}{ Variables } & \multicolumn{6}{|c|}{ Tick } \\
\hline & AL & B & BMO & BNS & BVI & CAE \\
\hline$E V O L_{j, t}$ & $\begin{array}{c}.1109 \\
(2.28)^{*}\end{array}$ & $\begin{array}{l}.1003 \\
(1.32)\end{array}$ & $\begin{array}{l}.1236 \\
(1.18)\end{array}$ & $\begin{array}{c}.2213 \\
(3.54)^{* *}\end{array}$ & $\begin{array}{c}.7428 \\
(1.45)\end{array}$ & $\begin{array}{l}1.3420 \\
(2.24)^{*}\end{array}$ \\
\hline$U V O L_{j, t}$ & $\begin{array}{c}.3209 \\
(7.43)^{* *}\end{array}$ & $\begin{array}{c}.0623 \\
(2.25)^{*}\end{array}$ & $\begin{array}{l}.0106 \\
(1.23)\end{array}$ & $\begin{array}{l}.1925 \\
(2.45)^{*}\end{array}$ & $\begin{array}{c}.4521 \\
(3.05)^{* *}\end{array}$ & $\begin{array}{c}.2054 \\
(2.30)^{*}\end{array}$ \\
\hline$N T_{j, t}$ & $\begin{array}{c}.7065 \\
(7.24)^{* *}\end{array}$ & $\begin{array}{c}.2154 \\
(6.75)^{* *}\end{array}$ & $\begin{array}{c}.4832 \\
(7.62)^{* *}\end{array}$ & $\begin{array}{c}.3621 \\
(5.31)^{* *}\end{array}$ & $\begin{array}{l}1.1248 \\
(3.34)^{* *}\end{array}$ & $\begin{array}{c}2.7223 \\
(7.54)^{* *}\end{array}$ \\
\hline$D U M_{j, t}$ & $\begin{array}{r}-.1876 \\
(-12.4)^{* *}\end{array}$ & $\begin{array}{l}-.0686 \\
(-1.76)\end{array}$ & $\begin{array}{c}-.0865 \\
(-7.65)^{* *}\end{array}$ & $\begin{array}{c}-.1264 \\
(-4.29)^{* *}\end{array}$ & $\begin{array}{c}-.1226 \\
(-9.87) * *\end{array}$ & $\begin{array}{c}-.2081 \\
(-7.43)^{* * *}\end{array}$ \\
\hline$S P D_{j, t-1}$ & $\begin{array}{c}.1667 \\
(4.29)^{* *}\end{array}$ & $\begin{array}{c}.2514 \\
(6.54)^{* *}\end{array}$ & $\begin{array}{c}.2112 \\
(5.45)^{* * *}\end{array}$ & $\begin{array}{c}.2927 \\
(6.71)^{* *}\end{array}$ & $\begin{array}{c}.0918 \\
(6.89)^{* *}\end{array}$ & $\begin{array}{c}.1154 \\
(3.97)^{* *}\end{array}$ \\
\hline$Q D_{j, t-1}$ & $\begin{array}{c}-1.3233 \\
(-4.98)^{* *}\end{array}$ & $\begin{array}{c}-.3982 \\
(-3.02)^{* * *}\end{array}$ & $\begin{array}{l}-.2092 \\
(-1.11)\end{array}$ & $\begin{array}{l}-1.1345 \\
(-4.78)^{* * *}\end{array}$ & $\begin{array}{l}-1.5463 \\
(-3.72)^{* *}\end{array}$ & $\begin{array}{c}-1.7623 \\
(-3.98)^{* * *}\end{array}$ \\
\hline $\begin{array}{l}O P_{t} \\
\sigma_{j, t-i}\end{array}$ & $\begin{array}{c}.1924 \\
(5.54)^{* *} \\
{[4.91]^{* *}}\end{array}$ & $\begin{array}{c}.2101 \\
(5.72)^{* *} \\
{[4.56]^{* *}}\end{array}$ & $\begin{array}{c}.1939 \\
(6.41)^{* *} \\
{[10.3]^{* *}}\end{array}$ & $\begin{array}{c}.3518 \\
(6.74)^{* *} \\
{[3.98]^{* *}}\end{array}$ & $\begin{array}{c}.3013 \\
(5.34)^{* *} \\
{[5.87]^{* *}}\end{array}$ & $\begin{array}{l}.1011 \\
(1.21) \\
{[6.32]^{* *}}\end{array}$ \\
\hline $\begin{array}{l}\mathrm{DW} \\
\mathrm{R}^{2}\end{array}$ & $\begin{array}{l}2.035 \\
.2323\end{array}$ & $\begin{array}{l}2.024 \\
.2504\end{array}$ & $\begin{array}{l}2.024 \\
.2723\end{array}$ & $\begin{array}{l}2.019 \\
.2109\end{array}$ & $\begin{array}{l}2.021 \\
.2982\end{array}$ & $\begin{array}{l}2.022 \\
.3109\end{array}$ \\
\hline
\end{tabular}


TABLE 3. (Continued)

\begin{tabular}{|c|c|c|c|c|c|c|}
\hline \multirow[b]{2}{*}{ Variables } & \multicolumn{6}{|c|}{ Tick } \\
\hline & $\mathrm{CM}$ & $\mathrm{CP}$ & CTR.A & $\mathrm{ECO}$ & GOU & IMO.A \\
\hline \multirow{2}{*}{$E V O L_{j, t}$} & .2045 & .1430 & .0699 & 1.0330 & .0201 & .6423 \\
\hline & $(2.98)^{* *}$ & $(1.23)$ & $(.50)$ & $(3.01)^{* *}$ & $(.76)$ & $(2.37)^{*}$ \\
\hline \multirow{2}{*}{$U V O L_{j, t}$} & .1965 & .0145 & .1754 & .7998 & .1265 & .5484 \\
\hline & $(4.87) * *$ & $(2.46)^{*}$ & $(4.01)^{* *}$ & $(4.25)^{* *}$ & $(1.34)$ & $(5.47)^{* *}$ \\
\hline \multirow[t]{2}{*}{$N T_{j, t}$} & .8796 & .3076 & 1.4921 & 2.6754 & 2.4351 & 1.0833 \\
\hline & $(7.45)^{* *}$ & $(9.78)^{* *}$ & $(6.32)^{* *}$ & $(7.31)^{* *}$ & $(5.45)^{* *}$ & $(4.56)^{* *}$ \\
\hline \multirow[t]{2}{*}{$D U M_{j, t}$} & -.2030 & -.1801 & -.1150 & -.2287 & -.1998 & -.0797 \\
\hline & $(-11.6)^{* *}$ & $(-4.95)^{* *}$ & $(-11.8)^{* *}$ & $(-10.8)^{* *}$ & $(-10.6)^{* *}$ & $(-8.72)^{* *}$ \\
\hline \multirow[t]{2}{*}{$S P D_{j, t-1}$} & .2432 & .2176 & .1345 & .1254 & .1411 & .1291 \\
\hline & $(8.53)^{* *}$ & $(7.01)^{* *}$ & $(6.89)^{* *}$ & $(6.54)^{* *}$ & $(5.54)^{* *}$ & $(3.76)^{* *}$ \\
\hline \multirow[t]{2}{*}{$Q D_{j, t-1}$} & -1.0892 & -.7834 & -1.7124 & -3.6325 & -2.7425 & -2.5343 \\
\hline & $(-3.71) * *$ & $(-3.95)^{* *}$ & $(-4.51)^{* *}$ & $(-3.92) * *$ & $(-3.76)^{* *}$ & $(-3.98)^{* *}$ \\
\hline \multirow[t]{2}{*}{$O P_{t}$} & .3212 & .1242 & .1254 & .8753 & .2333 & .2092 \\
\hline & $(4.56)^{* *}$ & $(3.87)^{* *}$ & $(4.65)^{* *}$ & $(8.09)^{* *}$ & $(4.55)^{* *}$ & $(6.71)^{* *}$ \\
\hline$\sigma_{j, t-i}$ & {$[5.87]^{* *}$} & {$[6.25]^{* *}$} & {$[5.76]^{* *}$} & {$[4.77] * *$} & {$[5.61]^{* *}$} & {$[6.18]^{* *}$} \\
\hline DW & 2.022 & 2.019 & 2.000 & 1.991 & 2.015 & 2.013 \\
\hline $\mathrm{R}^{2}$ & .2702 & .2453 & .1978 & .2989 & .2571 & .3109 \\
\hline
\end{tabular}

(Continued) 
TABLE 3. (Continued)

\begin{tabular}{|c|c|c|c|c|c|c|}
\hline \multirow[b]{2}{*}{ Variables } & \multicolumn{6}{|c|}{ Tick } \\
\hline & IMS & LAC & LDM.B & MB & MCL & $\mathrm{N}$ \\
\hline & (1.29) & $(2.38)^{*}$ & $(2.45)^{*}$ & $(3.44)^{* *}$ & (1.65) & $(.54)$ \\
\hline \multirow[t]{2}{*}{$U V O L_{j, t}$} & .2221 & .4433 & .0733 & .3925 & .2006 & .0051 \\
\hline & $(3.97)^{* *}$ & $(4.95)^{* *}$ & $(1.52)$ & $(4.29)^{* *}$ & $(4.98)^{* *}$ & $(2.45)^{*}$ \\
\hline \multirow[t]{2}{*}{$D U M_{j, t}$} & -.1301 & -.2505 & -.1443 & -.1972 & -.1691 & -.1119 \\
\hline & $(-12.3)^{* *}$ & $(-11.7)^{* *}$ & $(-4.65)^{* *}$ & $(-6.48)^{* *}$ & $(-10.9) * *$ & $(-13.0)^{* *}$ \\
\hline \multirow[t]{2}{*}{$S P D_{j, t-1}$} & .1431 & .1361 & .2881 & .1512 & .1431 & .1104 \\
\hline & $(3.46)^{* *}$ & $(4.57)^{* *}$ & $(4.32)^{* *}$ & $(6.99) * *$ & $(5.01)^{* *}$ & $(3.23)^{* *}$ \\
\hline \multirow[t]{2}{*}{$Q D_{j, t-1}$} & -1.5376 & -1.1722 & -.2035 & -1.4345 & -1.8381 & -1.7265 \\
\hline & $(-2.22)^{*}$ & $(-3.51)^{* *}$ & $(-.39)$ & $(-5.09) * *$ & $(-4.98)^{* *}$ & $(-4.79) * *$ \\
\hline$D W$ & 2.014 & 2.016 & 2.031 & 2.011 & 2.017 & 2.036 \\
\hline$R^{2}$ & .2376 & .3284 & .2985 & .2452 & .2325 & .3106 \\
\hline
\end{tabular}


TABLE 3. (Continued)

\begin{tabular}{|c|c|c|c|c|c|c|}
\hline \multirow[b]{2}{*}{ Variables } & \multicolumn{6}{|c|}{ Tick } \\
\hline & NA & NOR & NTL & NVA & PDG & POW \\
\hline \multirow[t]{2}{*}{$E V O L_{j, t}$} & .1654 & .3823 & .3565 & .2054 & .4455 & 1.5382 \\
\hline & (1.09) & $(2.47)^{*}$ & $(3.76)^{* *}$ & $(4.92)^{* *}$ & $(4.07)^{* *}$ & (1.92) \\
\hline \multirow{2}{*}{$U V O L_{j, t}$} & .2821 & .1830 & .4395 & .1928 & .3528 & .5565 \\
\hline & $(3.98)^{* *}$ & $(3.19)^{* *}$ & $(4.43)^{* *}$ & $(4.76)^{* *}$ & $(4.12)^{* *}$ & $(5.87)^{* *}$ \\
\hline \multirow[t]{2}{*}{$N T_{j, t}$} & 1.5423 & 1.5874 & 1.3183 & .7988 & .8994 & .8784 \\
\hline & $(7.59)^{* *}$ & $(8.78)^{* *}$ & $(5.64)^{* *}$ & $(6.76)^{* *}$ & $(5.28) * *$ & $(3.41)^{* *}$ \\
\hline \multirow[t]{2}{*}{$D U M_{j, t}$} & -.1974 & -.1404 & -.1306 & .0172 & -.1769 & -.0867 \\
\hline & $(-10.6)^{* *}$ & $(-10.8)^{* *}$ & $(-9.67)^{* *}$ & $(.10)$ & $(-9.08) * *$ & $(-11.6)^{* *}$ \\
\hline \multirow[t]{2}{*}{$S P D_{j, t-1}$} & .1678 & .1232 & .2233 & .2134 & .1235 & .1299 \\
\hline & $(7.83)^{* *}$ & $(4.56)^{* *}$ & $(4.56)^{* *}$ & $(8.78)^{* *}$ & $(4.89)^{* * *}$ & $(5.67)^{* *}$ \\
\hline \multirow[t]{2}{*}{$Q D_{j, t-1}$} & -1.2281 & -1.7274 & -2.5273 & -2.2332 & -1.5356 & -1.9873 \\
\hline & $(-4.32)^{* *}$ & $(-4.87)^{* *}$ & $(-5.87) * *$ & $(-7.31)^{* *}$ & $(-3.39) * *$ & $(-3.65)^{* *}$ \\
\hline \multirow[t]{2}{*}{$O P_{t}$} & .3021 & .2803 & .2346 & .1676 & .5113 & .1427 \\
\hline & $(5.63)^{* *}$ & $(5.64)^{* *}$ & $(5.43)^{* *}$ & $(2.98)^{* *}$ & $(6.54) * *$ & $(6.02)^{* *}$ \\
\hline$\sigma_{j, t-i}$ & {$[10.3]^{* *}$} & {$[7.54]^{* *}$} & {$[5.41]^{* *}$} & {$[3.85]^{* *}$} & {$[1.75]$} & {$[9.53]^{* *}$} \\
\hline DW & 2.011 & 2.031 & 2.054 & 2.024 & 2.017 & 2.035 \\
\hline $\mathrm{R}^{2}$ & .2330 & .2652 & .2842 & .2301 & .3543 & .2101 \\
\hline
\end{tabular}

(Continued) 
TABLE 3. (Continued)

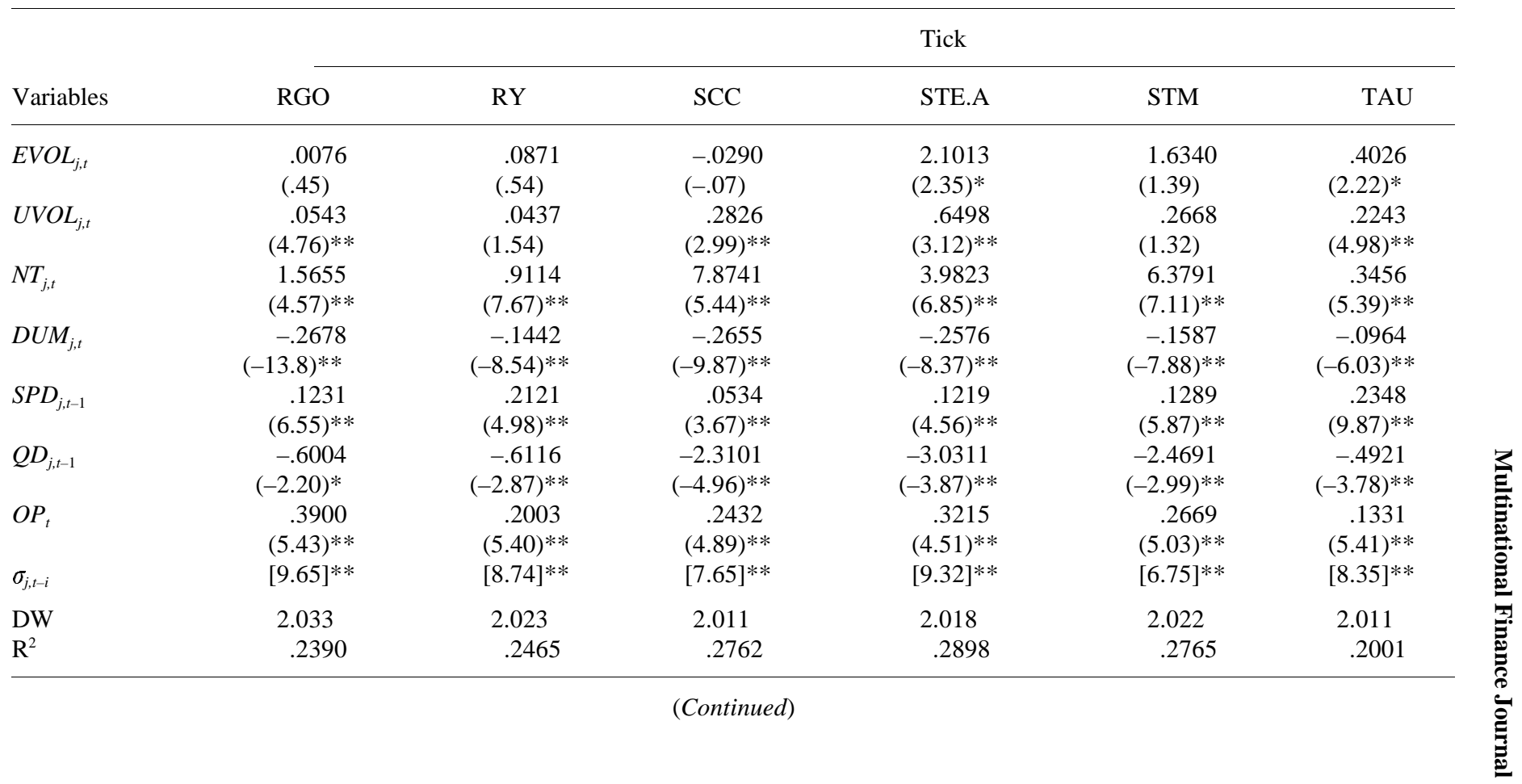


TABLE 3. (Continued)

\begin{tabular}{|c|c|c|c|c|c|c|}
\hline \multirow[b]{2}{*}{ Variables } & \\
\hline & TD & TEK.B & TOC & TRP & VO & TIPS \\
\hline \multirow{2}{*}{$E V O L_{j, t}$} & .1063 & .0511 & .1376 & .1234 & -.1043 & .1587 \\
\hline & (1.37) & $(.43)$ & $(.76)$ & (1.43) & $(-.65)$ & $(1.73)$ \\
\hline \multirow{2}{*}{$U V O L_{j, t}$} & .0800 & .3021 & .2443 & .2123 & .3226 & .4112 \\
\hline & (1.58) & $(4.53)^{* *}$ & $(5.40)^{* *}$ & $(1.39)$ & $(2.34)^{*}$ & $(1.75)$ \\
\hline \multirow[t]{2}{*}{$N T_{j, t}$} & .8001 & 2.7887 & 2.6775 & .5538 & 1.3668 & .9061 \\
\hline & $(6.76)^{* *}$ & $(5.76)^{* *}$ & $(13.0)^{* *}$ & $(3.79)^{* *}$ & $(9.89)^{* *}$ & $(2.31)^{*}$ \\
\hline \multirow[t]{2}{*}{$D U M_{j, t}$} & -.1175 & -.1587 & -.0766 & -.0549 & -.0578 & -.8239 \\
\hline & $(-6.47) * *$ & $(-8.39) * *$ & $(-5.87) * *$ & $(-4.67) * *$ & $(-10.9) * *$ & $(-8.03) * *$ \\
\hline \multirow{2}{*}{$S P D_{j, t-1}$} & .2249 & .1103 & .2101 & .2664 & .1105 & .2723 \\
\hline & $(5.19)^{* *}$ & $(5.59)^{* *}$ & $(8.45)^{* *}$ & $(8.32) * *$ & $(3.02)^{* *}$ & $(6.74)^{* *}$ \\
\hline \multirow[t]{2}{*}{$Q D_{j, t-1}$} & -.8272 & -1.4354 & -.7765 & -.8841 & -4.6574 & -.4628 \\
\hline & $(-3.89) * *$ & $(-4.31)^{* *}$ & $(-3.87) * *$ & $(-4.67)^{* *}$ & $(-2.11)^{*}$ & $(-3.67)^{* *}$ \\
\hline \multirow[t]{2}{*}{$O P_{t}$} & .3134 & .3217 & .1109 & .1271 & .1447 & .2291 \\
\hline & $(4.51)^{* *}$ & $(4.87)^{* *}$ & $(4.01)^{* *}$ & $(4.31) * *$ & $(6.09)^{* *}$ & $(6.71)^{* *}$ \\
\hline$\sigma_{j, t-i}$ & {$[7.42]^{* *}$} & {$[4.55]^{* *}$} & {$[6.48] * *$} & {$[2.40]^{*}$} & {$[7.64]^{* *}$} & {$[9.48]^{* *}$} \\
\hline DW & 2.023 & 2.075 & 2.012 & 1.989 & 2.003 & 2.010 \\
\hline $\mathrm{R}^{2}$ & .2376 & .2872 & .2590 & .2689 & .2540 & .1918 \\
\hline
\end{tabular}

Note: Regression of estimated volatility $\left(\sigma_{j, t}\right)$ on volume components and quote variables is given by: $\sigma_{j, t}=a_{j}+b_{j, 1} E V O L_{j, t}+b_{j, 2} U V O L_{j, t}+b_{i, 3} N T_{j, t}$ $+b_{j, 4} D U M_{j, t}+b_{j, 5} S P D_{j, t-1}+b_{j, 6} Q D_{j, t-1}+b_{j, 7} O P_{t}+\Sigma c_{j, i} \sigma_{j, t-1}+e_{j, t}$, where $E V O L_{j t}$ is expected volume; $U V O L_{j t}$ is unexpected volume; $N T_{j t}$ is number of trades, $D U M_{j t}$ is the indicator variable for no trade outcomes, $S P D_{j, t-1}$ is the percent quoted spread, $Q D_{j, t-1}$ is quote depth defined as the sum of bid and ask depths, and $O P_{t}$ is the indicator variable for opening intervals. Durbin-Watson (DW) statistics and adjusted $\mathrm{R}^{2}$ are provided. An ARMA(3,3) with a seasonal adjustment model is used to partition volume into expected and unexpected components. The dependent variable is estimated volatility based on the IWLS procedure used by Schwert (1990). $*(*)$ denotes statistical significance at 5\% (1\%) level. Test statistics for individual coefficients are on the IWLS procedure used by Schwert (1990). $*(* *)$ denotes statistical significance at $5 \%(1 \%)$ level. Test statistics for individual coefficients are
t-statistics (in parentheses) computed using White (1980) standard errors. Test statistics for lagged volatility coefficients are F-statistics (in brackets) for the hypothesis that the sum of the 13 coefficients is zero. 
slightly higher than those for Regression 5 for most of the stocks. A regression without the number of trades is also estimated but, to save space, not reported. Coefficients for unexpected volume are significant for 24 of the 35 stocks, but the adjusted R-squares are smaller than those with number of trades. This suggests that including unexpected volume alone is not sufficient to capture the information conveyed by number of trades.

The observed weak volume-volatility relationship can be explained by the role of expected volume arising mostly from liquidity trading. Discretionary uninformed traders tend to concentrate their trading to reduce adverse information costs (Admati and Pfleiderer [1988]). Thus, part of expected volume is likely to reduce volatility. However, total volume also incorporates the volume of anticipated informed trading based on recent trading activities. Order types tend to depend positively on previous orders, partly due to the process of working an order by traders over time (Hasbrouck and Ho [1987]). Therefore, trading volume tends to capture the net (and ambiguous) effect.

\section{Concluding Remarks}

In this article, the dynamic relationships of volatility with a number of trading activity and quote variables are investigated by using half-hour data obtained from the intraday data for the TSE. The regression analysis indicates that the relationship between volume and volatility is weak and ambiguous when volatility is conditioned on number of trades and measures of quoted liquidity. In addition, the asymmetric response of volatility to seller-initiated volume is not evident. The weak volumevolatility relationship suggests volume reflects a net effect of both information flow and supply of market liquidity.

Volume is further partitioned into expected and unexpected components through an ARMA model, and unexpected volume is found to have a significantly positive relationship with volatility. However, the incremental explanatory power is stronger for trade frequency than for unexpected volume.

Thus, the role of volume as a proxy for information flow is subsumed by trade frequency. Number of trades is an alternative way of decomposing trading volume, and our results show a consistently positive relationship between number of trades and volatility for individual stocks and the traded market index. This suggests that 
number of trades may be a more effective proxy for the rate of information flow. Non-trade outcomes are significantly negatively related to volatility, consistent with prior research that such outcomes serve as a signal for a lack of information. Measures of quoted liquidity are also associated significantly with volatility and should be included in the volatility prediction model. The relationship is positive for quoted spreads and negative for quoted depth.

\section{Appendix}

The names and corresponding tick symbols for the 35 firms included in the TSE 35 Index as of June 1990 are as follows:

Company Name

Alcan Aluminium

BCE Inc.

Bank of Montreal

Bank of Nova Scotia

Bow Valley Industrials

CAE Industrials

Canadian Imperial Bank

Canadian Pacific Ltd.

Canadian Tire Class A

Echo Bay Mines

Gulf Canada Resources

Imperial Oil Class A

Imasco Ltd.

Lac Minerals

Laidlaw Class B

Macmillan Bloedel

Moore Corp.

Inco Ltd.

National Bank

Noranda Inc.

Northern Telecom

Nova Corp. of Alberta

Placer Dome

Power Corp.

Ranger Oil

Royal Bank

Sears Canada
Tick Symbol

AL
B
BMO
BNS
BVI
CAE
CM
CP
CTR.A
ECO
GOU
IMO.A
IMS
LAC
LDM.B
MB
MCL
N
NA
NOR
NTL
NVA
PDG
POW
RGO
RY
SCC

BNS

BVI

$\mathrm{CAE}$

$\mathrm{CP}$

CTR.A

ECO

MO.A

MS

DM.B

$\mathrm{MB}$

MCL

$\mathrm{N}$

NOR

NTL

PDG

POW

SCC 
Stelco Series A $\quad$ STE.A

Southam Inc. $\quad$ STM

TransAlta Utilities TAU

Toronto-Dominion Bank TD

Teck Corp. Class B TEK.B

Thomson Corp. TOC

TransCanada Pipelines $\quad$ TRP

Seagram Co. VO

\section{References}

Admati, A.R., and Pfleiderer, P. 1988. A theory of intraday patterns: Volume and price variability. Review of Financial Studies 1: 3-40.

Barclay, M. J., and Warner, J. B. 1993. Stealth trading and volatility: Which trades move prices. Journal of Financial Economics 34: 281-305.

Bessembinder, H., and Seguin, P. 1992. Future-trading activity and stock price volatility. Journal of Finance 47: 2015-2034.

Bessembinder, H., and Seguin, P. 1993. Price volatility, trading volume, and market depth: Evidence from futures markets. Journal of Financial and Quantitative Analysis 28: 21-39.

Chan, L. K. C., and Lakonishok, J. 1993. Institutional trades and intraday stock price behavior. Journal of Financial Economics 33: 173-199.

Clark, P. K. 1973. A subordinated stochastic process model with finite variances for speculative prices. Econometrica 41: 135-155.

Davidian, M., and Carroll, R. J. 1987. Variance function estimation. Journal of the American Statistical Association 82: 1079-1091.

Easley, D., and O'Hara, M. 1987. Price, trade size, and information in securities markets. Journal of Financial Economics 19: 69-90.

Easley, D., and O'Hara, M. 1992. Time and the process of security price adjustment. Journal of Finance 47: 577-605.

Engle, R. F., and Bollerslev, T. 1986. Modelling the persistence of conditional variances. Econometric Reviews 5: 1-50.

Gallant, A. R.; Rossi, P. E.; and Tauchen, G. 1992. Stock prices and volume. Review of Financial Studies 5: 199-242.

George, T. J.; Kaul, G.; and Nimalendran, M. 1994. Trading volume and transaction costs in specialist markets. Journal of Finance 49: 1489-1505.

Glosten, L. R., and Milgrom, P. R. 1985. Bid, ask and transaction prices in a specialist market with heterogeneously informed traders. Journal of Financial Economics 14: 71-100.

Harris, L. 1987. Transaction data tests of the mixture of distributions hypothesis. Journal of Financial and Quantitative Analysis 22: 127-141.

Hasbrouck, J., and Ho, T. 1987. Order arrival, quote behavior, and returngenerating process. Journal of Finance 42: 1035-1048. 
Jain, P. C., and Joh, G.-H. 1988. The dependence between hourly prices and trading volume. Journal of Financial and Quantitative Analysis 23: 269283.

Jones, C. M.; Kaul, G.; and Lipson, M. L. 1994. Transactions, volume, and volatility. Review of Financial Studies 36: 127-154.

Karpoff, J. 1987. The relation between price changes and trading volume: A survey, Journal of Financial and Quantitative Analysis 22: 109-126.

Kaul, G., and Nimalendran, M. 1990. Price reversals: Bid-ask errors or market overreaction? Journal of Financial Economics 28: 67-93.

Kyle, A. S. 1985. Continuous auctions and insider trading. Econometrica 53: 1315-1335.

Lamoureux, C. G., and Lastrapes, W. D. 1990. Heteroscedasticity in stock return data: Volume versus GARCH effects. Journal of Finance 45: 221230.

Lee, C. M. C.; Mucklow, B.; and Ready, M. J. 1993. Spreads, depths, and the impact of earnings information: An intraday analysis. Review of Financial Studies 6: 345-374.

Madhavan, A. 1992. Trading mechanisms in securities markets. Journal of Finance 47: 607-641.

Mitchell, M. L., and Mulherin, J. H. 1994. The impact of public information on the stock market. Journal of Finance 49: 923-950.

Pagan, A. 1984. Econometric issues in the analysis of regressions with generated regressors. International Economic Review 25: 221-247.

Schwert, G. W. 1989. Why does stock market volatility change over time? Journal of Finance 44: 1115-1153.

Schwert, G. W. 1990. Stock volatility and the crash of '87. Review of Financial Studies 3: 77-102.

Stoll, H. R., and Whaley, R. E. 1990. The dynamics of stock index and stock index futures returns. Journal of Financial and Quantitative Analysis 25: 441-468.

Tauchen, G., and Pitts, M. 1983. The price variability-volume relationship on speculative markets. Econometrica 51: 485-505.

White, H. 1980. A heteroscedaticity-consistent covariance matrix estimator and a direct test for heteroscedasticity. Econometrica 48: 817-838. 